\title{
Torsion Pairs in Triangulated Categories
}

\author{
Chunyan Fan, Hailou Yao \\ College of Applied Sciences, Beijing University of Technology, Beijing, China \\ Email: fanchunyan@emails.bjut.edu.cn
}

Received February 28, 2013; March 30, 2013; accepted April 26, 2013

Copyright (C) 2013 Chunyan Fan, Hailou Yao. This is an open access article distributed under the Creative Commons Attribution License, which permits unrestricted use, distribution, and reproduction in any medium, provided the original work is properly cited.

\begin{abstract}
We study the properties of torsion pairs in triangulated category $\mathfrak{C}$ by introducing the notions of d-Ext-projectivity and d-Ext-injectivity. In terms of $\mathfrak{D}$-mutation of torsion pairs, we investigate the properties of torsion pairs in triangulated category $\mathfrak{U}=\mathfrak{Z} /[\mathfrak{D}]$ under some conditions on subcategories $\mathfrak{Z}$ and $\mathfrak{D}$ in $\mathfrak{C}$.
\end{abstract}

Keywords: d-Ext-Projectivity (d-Ext-Injectivity); Torsion Pairs; $\mathfrak{D}$-Mutation; Triangulated Category

\section{Introduction}

The notion of torsion theory (torsion pairs) in abelian categories was introduced by Dickson in 1966. Torsion theory plays an important role in the investigation of an abelian category. An abelian category is naturally embedded in a triangulated category like the bounded derived category. The analogous definition of torsion pairs in triangulated category is closely related to the notion of a t-structure. Beilinson, Bernstein and Deligne [1] introduce the definition of a t-structure in a triangulated category $\mathfrak{C}$.The t-structure is a pair $\left(\mathfrak{T}^{\leq 0}, \mathfrak{T}^{\geq 0}\right)$ of full subcategories such that setting $\mathfrak{T}^{\leq n}=\Sigma^{-n}\left(\mathfrak{T}^{\leq 0}\right)$ and $\mathfrak{T}^{\geq n}=\Sigma^{-n}\left(\mathfrak{T}^{\geq 0}\right), \forall n \in \mathbb{Z}$, satisfying:

$\mathfrak{C}\left(\mathfrak{T}^{\leq 0}, \mathfrak{T}^{\geq 0}\right)=0 ; \mathfrak{T}^{\leq 0} \subseteq \mathfrak{T}^{\leq 1} ; \mathfrak{T}^{\geq 1} \subseteq \mathfrak{T}^{\geq 0} ;$ any object

$\mathrm{C} \in \mathfrak{C}$ is included in a triangle

$$
C^{\leq 0} \rightarrow C \rightarrow C^{\geq 1} \rightarrow \Sigma\left(C^{\leq 0}\right),
$$

where $C^{\leq 0} \in \mathfrak{T}^{\leq 0}$, and $C^{\geq 1} \in \mathfrak{T}^{\geq 1}$. In [2], Beligiannis and Reiten studied the torsion theory on pretriangulated, triangulated and stable categories. They discussed the connection between torsion theories in abelian and derived categories and indicated the relationship with tilting theory, they point out that the torsion pairs in triangulated category and t-structures essentially coincide. In 1987, Gorodentsev and Rudakov [3] made use of mutation when they classified the exceptional vector bundles on $\mathfrak{P}^{2}$ where $\mathfrak{P}$ is a projective space. Mutation can be regarded as a categorical realization of Coxeter or braid groups. In [4] and [5], Fomin and Zelevinsky introduced cluster algebras, these algebras give an alge- braic and combinational framework for the positivity and canonical basis of quantum groups, which enjoy important combinational properties given in terms of the mutation for skew symmetric matrices. Cluster categories were introduced in [6], in which the mutation of cluster tilting objects was introduced. Recently, Geiss, Leclerc and Schroer [7] applied mutation to study rigid modules over preprojective algebras and the coordinate rings of maximal unipotent subgroups of semisimple Lie groups. Later Iyama and Yoshino [8] introduced the mutation of n-cluster tilting subcategories based on approximation theory. Recently, Zhou and Zhu [9] studied the notion of $\mathfrak{D}$-mutation of torsion pairs in triangulated categories, and they proved that the $\mathfrak{D}$-mutation of torsion pairs in triangulated categories is a torsion pair.They also studied its geometric meaning when the triangulated categories are the cluster categories of type $A_{n}$ or $A_{\infty}$.

In this paper, we study the torsion pairs in triangulated categories and their properties in terms of $\mathfrak{D}$-mutation pair. In a fixed triangulated category $\mathfrak{C}$, we give the definition of torsion pairs in $\mathfrak{C}$ and study their properties with the notion of subcategory $\mathfrak{P}(\mathfrak{X})$ (resp. $\mathfrak{I}(\mathfrak{X})$ ) whose objects are d-Ext-projective (resp. d-Extinjective). Under reasonable conditions on subcategories $\mathfrak{D}$ and $\mathfrak{Z}$ of $\mathfrak{C}$, we study the properties of torsion pairs in triangulated category $\mathfrak{Z} / \mathfrak{D}$ in terms of $\mathfrak{D}$ mutation pair.

\section{Preliminaries}

Through this paper, let $\mathfrak{C}$ be a triangulated category. We introduce some basic notions which will be used. Let $\mathfrak{X}$ and $\mathfrak{Y}$ be subcategories of $\mathfrak{C}$. We put 
$\mathfrak{X}^{\perp}=\left\{M \in \mathfrak{C} \mid \operatorname{Hom}_{\mathfrak{C}}(\mathfrak{X}, M)=0\right\}$

and ${ }^{\perp} \mathfrak{X}=\left\{N \in \mathfrak{C} \mid \operatorname{Hom}_{\mathfrak{C}}(N, \mathfrak{X})=0\right\}$.

We denote by $\mathfrak{X} * \mathfrak{Y}$ the collection of objects in $\mathfrak{X}$ consisting of all such $\mathrm{M} \in \mathfrak{C}$ with the triangle

$$
X \stackrel{f}{\longrightarrow} M \stackrel{g}{\longrightarrow} Y \stackrel{h}{\longrightarrow} X[1]
$$

where $X \in \mathfrak{X}, Y \in \mathfrak{Y}$ By the octahedralaxiom, we have $(\mathfrak{X} * \mathfrak{Y}) * \mathfrak{Z}=\mathfrak{X} *(\mathfrak{Y} * \mathfrak{Z})$.

Definition 2.1 We call a pair $(\mathfrak{X}, \mathfrak{Y})$ of subcategories of $\mathfrak{C}$ a torsion pair if $\operatorname{Hom}_{\mathfrak{C}}(\mathfrak{X}, \mathfrak{Y})=0$ and

$\mathfrak{C}=\mathfrak{X} * \mathfrak{Y}$.

In this case, we can see that $\mathfrak{X}={ }^{\perp} \mathfrak{Y}$ and $\mathfrak{Y}=\mathfrak{X}^{\perp}$.

Let $f \in \operatorname{Hom}_{\mathfrak{C}}(\mathfrak{X}, \mathfrak{Y})$ be a morphism, we call $f$ a right $\mathfrak{X}$-approximation of $Y \in \mathfrak{C} \quad$ [10] if $X \in \mathfrak{X}$ and

$$
\operatorname{Hom}_{\mathfrak{C}}(-, X) \stackrel{\operatorname{Hom}_{\mathfrak{C}}(-, f)}{\longrightarrow} \operatorname{Hom}_{\mathfrak{C}}(-, Y) \rightarrow 0
$$

is exact as functors on $\mathfrak{X}$. We call $\mathfrak{X}$ a contravariantly finite subcategory of $\mathfrak{C}$ if any $Y \in \mathfrak{C}$ has a right $\mathfrak{X}$ approximation. Dually, for a morphism

$g \in \operatorname{Hom}_{C}(X, Y)$, we call $g$ a left $\mathfrak{Y}$-approximation of $X \in \mathfrak{C}$ if $Y \in \mathfrak{Y}$ and

$\operatorname{Hom}_{\mathfrak{C}}(Y,-) \stackrel{\operatorname{Hom}_{\mathfrak{C}}(g,-)}{\longrightarrow} \operatorname{Hom}_{\mathfrak{C}}(X,-) \rightarrow 0$ is exact. We call $Y$ a covariantly finite subcategory of $\mathfrak{C}$ if any $X \in \mathfrak{C}$ has a left $\mathfrak{Y}$-approximation.

Let $\mathfrak{D}$ be a subcategory of $\mathfrak{C}$, we call $h \in \operatorname{Hom}_{\mathfrak{C}}(X, Y) \mathfrak{D}$-monic (resp. $\mathfrak{D}$-epic) if $\operatorname{Hom}_{\mathfrak{C}}(Y, \mathfrak{D}) \rightarrow \operatorname{Hom}_{\mathfrak{C}}(X, \mathfrak{D}) \rightarrow 0$ (resp. $\left.\operatorname{Hom}_{\mathfrak{C}}(\mathfrak{D}, X) \rightarrow \operatorname{Hom}_{\mathfrak{C}}(\mathfrak{D}, Y) \rightarrow 0\right)$ is exact.

\section{Torsion Pairs in Triangulated Categories}

In this section, we introduce $d$-cluster tilting torsion pairs, rigid torsion pairs and maximal rigid torsion pairs in a triangulated category $\mathfrak{C}$, and study the properties of these torsion pairs.

Definition 3.1 Let $\mathfrak{X}$ be an extension-closed subcategory of $\mathfrak{C}$. An object $T \in \mathfrak{X}$ is called a d-Extprojective object of $\mathfrak{X}$ if $\operatorname{Ext}^{i}(T, \mathfrak{X})=0$ for all

$1 \leq i \leq d-1$. The d-Ext-injective objects of $\mathfrak{X}$ are defined dually. An object $T \in \mathfrak{X}$ is called a d-Ext-injective object of $\mathfrak{X}$ if $\operatorname{Ext}^{i}(\mathfrak{X}, T)=0$ for all $1 \leq i \leq d-1$. The subcategory of $\mathfrak{X}$ consisting of d-Ext-projective (or d-Ext-injective) objects in $\mathfrak{X}$ is denoted by $\mathfrak{P}(\mathfrak{X})$ ( $\mathfrak{I}(\mathfrak{X})$ respectively).

Definition 3.2 Let $\mathfrak{X}$ and $\mathfrak{Y}$ be subcategories of the triangulated category $\mathfrak{C}$.

1) The pair $(\mathfrak{X}, \mathfrak{Y})$ is called a $d$-cluster tilting torsion pair if $(\mathfrak{X}, \mathfrak{Y})$ is a torsion pair and satisfies the property: $\mathfrak{X}$ is functorially finite in $\mathfrak{C}$ and $M \in \mathfrak{X}$ if and only if $\operatorname{Ext}^{i}(\mathfrak{X}, M)=0$ for all $1 \leq i \leq d-1$ [11].

2) The pair $(\mathfrak{X}, \mathfrak{Y})$ is called a rigid torsion pair if $(\mathfrak{X}, \mathfrak{Y})$ is a torsion pair and $\operatorname{Ext}^{i}(\mathfrak{X}, \mathfrak{X})=0$ for all $1 \leq i \leq d-1$ [12].
3) The pair $(\mathfrak{X}, \mathfrak{Y})$ is called a maximal rigid torsion pair provided that $(\mathfrak{X}, \mathfrak{Y})$ is a torsion pair, $\mathfrak{X}$ is a rigid subcategory and satisfies the property:

if $\operatorname{Ext}^{i}\left(M \oplus X, M \oplus X^{\prime}\right)=0$ for any $X, X^{\prime} \in \mathfrak{X}$ and all $1 \leq i \leq d-1$, then $M \in \mathfrak{X}$. In this case, the subcategory $\mathfrak{X}$ is called a maximal rigid subcategory [13].

Corollary 3.3 A pair $(\mathfrak{X}, \mathfrak{Y})$ is a maximal rigid torsion pair if and only if $\mathfrak{X}[i] \subseteq \mathfrak{Y}$ for all $1 \leq i \leq d-1$ and for any rigid object $M$ in $\mathfrak{C}$, we have $M[i-1] \in \mathfrak{X}[i-1] * \mathfrak{X}[i]$ for all $1 \leq i \leq d-1$.

Proof: Now supposing $(\mathfrak{X}, \mathfrak{Y})$ is a maximal rigid torsion pair, by the definition we have

$\operatorname{Hom}(\mathfrak{X}, \mathfrak{Y})=0, \mathfrak{C}=\mathfrak{X} * \mathfrak{Y}$ and

$0=\operatorname{Ext}^{i}(\mathfrak{X}, \mathfrak{X})=\operatorname{Hom}(\mathfrak{X}, \mathfrak{X}[i])$ for all $1 \leq i \leq d-1$. It implies $\mathfrak{X}[i] \subseteq \mathfrak{Y}$ for all $1 \leq i \leq d-1$. For any rigid object $M$ in $\mathfrak{C}$, take a triangle

$$
X_{2} \rightarrow X_{1} \rightarrow M \rightarrow X_{2}[1]
$$

where $X_{1}, X_{2} \in \mathfrak{X}$. Then we have the triangle

$$
X_{2}[i-1] \rightarrow X_{1}[i-1] \rightarrow M[i-1] \rightarrow X_{2}[i]
$$

It follows that $M[i-1] \in \mathfrak{X}[i-1] * \mathfrak{X}[i]$ for all $1 \leq i \leq d-1$.

Conversely, suppose $(\mathfrak{X}, \mathfrak{Y})$ is a torsion pair with $\mathfrak{X}[i] \subseteq \mathfrak{Y}$ for all $1 \leq i \leq d-1$ and

$M[i-1] \in \mathfrak{X}[i-1] * \mathfrak{X}[i]$ for any rigid object $M$ while $1 \leq i \leq d-1$, then $\mathfrak{X}$ is rigid. If there exists an object $M$ in $\mathfrak{C}$ such that $\operatorname{Ext}^{i}\left(M \oplus X_{1}, M \oplus X_{2}\right)=0$ for any $X_{1}, X_{2} \in \mathfrak{X}$ and all $1 \leq i \leq d-1$. Then $M$ is rigid. It follows that there is a triangle

$$
X[i-1] \rightarrow M[i-1] \rightarrow X^{\prime}[i-1] \rightarrow X[i]
$$

for $X_{1}, X_{2} \in \mathfrak{X}$.

Then the above triangle splits. This implies that

$$
M[i-1] \cong X[i-1] \oplus X^{\prime}[i-1]
$$

for $1 \leq i \leq d-1$, i.e., $M \in \mathfrak{X}$. $\mathfrak{X}$ is maximal rigid.

Corollary 3.4 A pair $(\mathfrak{X}, \mathfrak{Y})$ is a $d$-cluster tilting torsion pair if and only if $\mathfrak{X}$ is functorially finite and $\mathfrak{X}[i]=\mathfrak{Y}$ for all $1 \leq i \leq d-1$.

Proof: By the definition, if we have

$0=\operatorname{Ext}^{i}(\mathfrak{X}, \mathfrak{X})=\operatorname{Hom}(\mathfrak{X}, \mathfrak{X}[i])$ for all $1 \leq i \leq d-1$, then we obtain $\mathfrak{X}[i]=\mathfrak{Y}$. On the other hand, $0=\operatorname{Hom}(\mathfrak{X}, \mathfrak{Y})=E x t^{i}(\mathfrak{X}, \mathfrak{Y}[-i])$ for all $1 \leq i \leq d-1$, it implys $\mathfrak{Y}[-i] \subseteq \mathfrak{X}$, i.e, $\mathfrak{Y} \subseteq \mathfrak{X}[i]$. So $\mathfrak{X}[i]=\mathfrak{Y}$.

Conversely, we only need to prove that $M \in \mathfrak{X}$ if and only if $\operatorname{Ext}^{i}(\mathfrak{X}, M)=0$ for all $1 \leq i \leq d-1$. Supposing $M \in \mathfrak{X}$, we have

$\operatorname{Ext}^{i}(\mathfrak{X}, M)=\operatorname{Hom}(\mathfrak{X}, M[i]) \subseteq \operatorname{Hom}(\mathfrak{X}, \mathfrak{Y})=0$ for

$1 \leq i \leq d-1$. Now, if $\operatorname{Ext}^{i}(\mathfrak{X}, M)=0$ for $1 \leq i \leq d-1$, we have that $M[i] \in \mathfrak{Y}=\mathfrak{X}[i]$, since 
$0=\operatorname{Ext}^{i}(\mathfrak{X}, M)=\operatorname{Hom}(\mathfrak{X}, M[i])$. This implies $M \in \mathfrak{X}$.

Proposition 3.5 Let $(\mathfrak{X}, \mathfrak{Y})$ be a rigid torsion pair, then $\mathfrak{I}(\mathfrak{X})=\mathfrak{X} \cap \mathfrak{Y}[-i], \mathfrak{P}(\mathfrak{Y})=\mathfrak{I}(\mathfrak{X})[i]$ for all

$1 \leq i \leq d-1$. Moreover, $\mathfrak{I}(\mathfrak{X})$ is covariantly finite in $\mathfrak{X}$ and $\mathfrak{P}(\mathfrak{Y})$ is contravariantly finite in $\mathfrak{Y}$.

Proof: Let $T \in \mathfrak{X}$, we have that

$0=\operatorname{Ext}^{i}(\mathfrak{X}, T)=\operatorname{Hom}(\mathfrak{X}, T[i])$ for $1 \leq i \leq d-1$ if and only if $T[i] \in \mathfrak{Y}$. Then $T \in \mathfrak{J}(\mathfrak{X})$ if and only if

$T \in \mathfrak{X} \cap \mathfrak{Y}[-i]$. For $\mathfrak{P}(\mathfrak{Y})$, let $M \in \mathfrak{Y}$,

$0=\operatorname{Ext}^{i}(M, \mathfrak{Y})=\operatorname{Hom}(M[-i], \mathfrak{Y})$ if and only if

$M[-i] \in \mathfrak{X}$. Since $M[-i] \in \mathfrak{Y}[-i]$, we have that

$M \in \mathfrak{P}(\mathfrak{Y})$ if and only if $M[-i] \in \mathfrak{X} \cap \mathfrak{Y}[-i]=\mathfrak{I}(\mathfrak{X})$, i.e., if and only if $M \in \Im(\mathfrak{X})[i]$.

Now we prove that $\mathfrak{I}(\mathfrak{X})$ is covariantly finite in $\mathfrak{X}$. Since $(\mathfrak{X}, \mathfrak{Y})$ is a torsion pair, we have that $(\mathfrak{X}[-i], \mathfrak{Y}[-i])$ is a torsion pair for $1 \leq i \leq d-1$. For any object $X \in \mathfrak{X}$, take a triangle

$$
X_{1}[-i] \rightarrow X \stackrel{h}{\longrightarrow} Y[-i] \rightarrow X_{1}[-i+1]
$$

where $X_{1} \in \mathfrak{X}, Y \in \mathfrak{Y}$. Then we have a triangle

$$
X \stackrel{h}{\longrightarrow} Y[-i] \rightarrow X_{1}[-i+1] \rightarrow X[1] .
$$

When $1 \leq i \leq d-1$, then

$\operatorname{Hom}\left(X_{1}[-i+1], \mathfrak{X}\right)=\operatorname{Ext}^{i-1}\left(X_{1}, \mathfrak{X}\right)=0$. Applying functor $\operatorname{Hom}(-, \mathfrak{X})$ to the triangle above, we obtain

$Y[-i] \in \mathfrak{X}$. Since $Y[-i] \in \mathfrak{Y}[-i]$, we have

$Y[-i] \in \mathfrak{I}(\mathfrak{X})$. Then $h$ is a left $\mathfrak{I}(\mathfrak{X})$-approximation of $\mathfrak{X}$. Thus $\mathfrak{I}(\mathfrak{X})$ is covariantly finite in $\mathfrak{X}$.

When $i=1$, we have that $\mathfrak{I}(\mathfrak{X})=\mathfrak{X} \cap \mathfrak{Y}[-1]$. For any $X \in \mathfrak{X}$, take a triangle

$$
X_{1}[-1] \rightarrow X \stackrel{h}{\longrightarrow} Y[-1] \rightarrow X_{1}
$$

where $X_{1} \in \mathfrak{X}$ and $Y \in \mathfrak{Y}$. Then we have the triangle

$$
X \stackrel{h}{\longrightarrow} Y[-1] \rightarrow X_{1} \rightarrow X[1]
$$

Since $\mathfrak{X}$ is extension-closed, we obtain that $Y[-1] \in \mathfrak{X}$, and hence $Y[-1] \in \mathfrak{I}(\mathfrak{X})$. It is easy to see that $\mathfrak{I}(\mathfrak{X})$ is covariantly finite in $\mathfrak{X}$.

Finally, we prove $\mathfrak{P}(\mathfrak{Y})=\mathfrak{I}(\mathfrak{X})[i]$ is contravariantly finite in $\mathfrak{Y}$. In case $i=1$, we have

$$
\mathfrak{P}(\mathfrak{Y})=\mathfrak{I}(\mathfrak{X})[1]=\mathfrak{X}[1] \cap \mathfrak{Y} .
$$

Since $(\mathfrak{X}, \mathfrak{Y})$ is a torsion pair, for any $Y \in \mathfrak{Y}$, there exists a triangle

$$
Y_{1} \rightarrow X_{1}[1] \stackrel{f}{\longrightarrow} Y \rightarrow Y_{1}[1]
$$

where $X_{1} \in \mathfrak{X}$ and $Y_{1} \in \mathfrak{Y}$. Since $X_{1}[1] \in \mathfrak{X}$ and $Y$ is closed under extensions, we have $X_{1}[1] \in \mathfrak{Y}$, and hence $X_{1}[1] \in \mathfrak{I}(\mathfrak{X})[1]=\mathfrak{P}(\mathfrak{Y})$. It follows that $f$ is a right $\mathfrak{P}(\mathfrak{Y})$-approximation of $\mathfrak{Y}$, and then $\mathfrak{P}(\mathfrak{Y})$ is contravariantly finite in $\mathfrak{Y}$.

In case $2 \leq i \leq d-1$, we have $\mathfrak{P}(\mathfrak{Y})=\mathfrak{I}(\mathfrak{X})[i]=\mathfrak{X}[i] \cap \mathfrak{Y}$ for and $Y \in \mathfrak{Y}$. Take a triangle

$$
Y_{1}[i-1] \rightarrow X_{1}[i] \stackrel{f}{\longrightarrow} Y \rightarrow Y_{1}[i]
$$

where $X_{1} \in \mathfrak{X}$ and $Y_{1} \in \mathfrak{Y}$. Since $X_{1}[i] \in \mathfrak{X}[i]$ and $\operatorname{Hom}\left(X, X_{1}[i]\right)=\operatorname{Ext}^{i}\left(X, X_{1}\right)=0$ for any $X \in \mathfrak{X}$ and $2 \leq i \leq d-1$, we have that $X_{1}[i] \in \mathfrak{Y}$. Hence

$X_{1}[i] \in \mathfrak{P}(\mathfrak{Y})$, i.e., $f$ is a right $\mathfrak{P}(\mathfrak{Y})$-approximation of $\mathfrak{Y}$. It means that $\mathfrak{P}(\mathfrak{Y})$ is contravariantly finite in $\mathfrak{Y}$.

Corollary $3.6(\mathfrak{X}, \mathfrak{Y})$ is a rigid torsion pair if and only if $\mathfrak{I}(\mathfrak{X})=\mathfrak{X}$.

Proof: By proposition 3.5, we have that $\mathfrak{I}(\mathfrak{X})=\mathfrak{X} \cap \mathfrak{Y}[-i]$. Since

$0=\operatorname{Ext}^{i}(\mathfrak{X}, \mathfrak{X})=\operatorname{Hom}(\mathfrak{X}, \mathfrak{X}[i])$ for all $1 \leq i \leq d-1$ if and only if $\mathfrak{X}[i] \subseteq \mathfrak{Y}$ for $1 \leq i \leq d-1$, hence in this case, $\mathfrak{I}(\mathfrak{X})=\mathfrak{X}$.

Corollary 3.7 Let $\mathfrak{X}$ be a maximal rigid subcategory of $\mathfrak{C}$, then

1) Every object $M \in \mathfrak{X}$ is d-Ext-projective (or d-Extinjective) in $\mathfrak{X}$.

2) An object $N \in \mathfrak{Y}$ is d-Ext-projective in $\mathfrak{Y}$ if and only if $N[-i] \in \mathfrak{X}$.

Proof: 1) By Corollary 3.6, $\mathfrak{I}(\mathfrak{X})=\mathfrak{X}$, (a) holds.

2) For any object $N \in \mathfrak{Y}$

$$
0=\operatorname{Ext}^{i}(N, \mathfrak{Y})=\operatorname{Hom}(N, \mathfrak{Y}[i])=\operatorname{Hom}(N[-i], \mathfrak{Y})
$$

if and only if $N[-i] \in \mathfrak{X}$.

\section{Torsion Pairs in $\mathfrak{U}$}

Let $\mathfrak{C}$ be a triangulated category and $\mathfrak{D}$ a subcategory of $\mathfrak{C}$ satisfying $\mathfrak{C}(\mathfrak{D}, \mathfrak{D}[1])=0$. For a subcategory $\mathfrak{X}$ of $\mathfrak{C}$, put $\mu^{-1}(\mathfrak{X} ; \mathfrak{D})=(\mathfrak{D} * \mathfrak{X}[1]) \cap^{\perp} \mathfrak{D}[1]$. Then $\mu^{-1}(\mathfrak{X} ; \mathfrak{D})$ consists of all $N \in \mathfrak{C}$ such that there exists a triangle

$$
X \stackrel{\alpha}{\longrightarrow} D \rightarrow N \rightarrow X[1]
$$

with $X \in \mathfrak{X}$ and a left $\mathfrak{D}$-approximation $\alpha$.

Dually, for a subcategory $\mathfrak{Y}$ of $\mathfrak{C}$, put

$$
\mu(\mathfrak{Y} ; \mathfrak{D})=(\mathfrak{Y}[-1] * \mathfrak{D}) D[-1]^{\perp} .
$$

Then $\mu(\mathfrak{Y} ; \mathfrak{D})$ consists of all $M \in \mathfrak{C}$ such that there exists a triangle $M \rightarrow D \stackrel{\beta}{\longrightarrow} Y \rightarrow M[1]$ with $Y \in \mathfrak{Y}$ and a right $\mathfrak{D}$-approximation $\beta$.

We call a pair $(\mathfrak{X}, \mathfrak{Y})$ of subcategories of $\mathfrak{C}$ a $\mathfrak{D}$ mutation pair [5] if $\mathfrak{D} \subset \mathfrak{Y} \subset \mu^{-1}(\mathfrak{X} ; \mathfrak{D})$ and $\mathfrak{D} \subset \mathfrak{X} \subset \mu(\mathfrak{Y} ; \mathfrak{D})$.

Let $\mathfrak{Z} \supset \mathfrak{D}$ be a subcategory of $\mathfrak{C}$, we assume:

1) $\mathfrak{Z}$ is extension closed; 
2) $(\mathfrak{Z}, \mathfrak{Z})$ forms a $\mathfrak{D}$-mutation pair.

In the rest of this section, we assume that $\mathfrak{C}$ has a serre functor $S$. We put $S_{n}=S \circ[-n]: \mathfrak{C} \rightarrow \mathfrak{C}$. We call a subcategory $\mathfrak{X}$ of $\mathfrak{C}$ an $S_{n}$-subcategory of $\mathfrak{C}$ if it satisfies $\mathfrak{X}=S_{n} \mathfrak{X}=S_{n}^{-1} \mathfrak{X}$.

For an integer $n \geq 1$, we call a subcategory $\mathfrak{M}$ of $\mathfrak{C} n$-rigid if $\mathfrak{C}(\mathfrak{M}, \mathfrak{M}[i])=0$ for $1 \leq i \leq n$.

Now we assume that $\mathfrak{D}$ is a functorially finite n-rigid subcategory of $\mathfrak{C}$ and

$$
\mathfrak{D} \subset \mathfrak{Z}=\bigcap_{i=1}^{n-1} \mathfrak{D}[-i]^{\perp}=\bigcap_{i=1}^{n-1} \perp \mathfrak{D}[i] .
$$

It was proved in [8] that $\mathfrak{U}=\mathfrak{Z} /[\mathfrak{D}]$ forms a triangulated category. The shift in $\mathfrak{U}$ is defined as follows: for any object $X \in \mathfrak{Z}$, consider the left $\mathfrak{D}$-approximation $f: X \rightarrow D$, and extend it to a triangle

$$
X \stackrel{f}{\longrightarrow} D \stackrel{g}{\longrightarrow} X\langle 1\rangle \stackrel{h}{\longrightarrow} X[1]
$$

where $D \in \mathfrak{D}$ and $X\langle 1\rangle \in \mathfrak{Z}$. The $X\langle 1\rangle$ is defined as the shift of $\mathfrak{X}$ in $\mathfrak{U}$.

Then triangles in $\mathfrak{U}$ are defined as the complex

$$
X \stackrel{\bar{a}}{\longrightarrow} Y \stackrel{\bar{b}}{\longrightarrow} Z \stackrel{\bar{c}}{\longrightarrow} X\langle 1\rangle
$$

in $\mathfrak{U}$, where $X, Y, Z \in \mathfrak{Z}$ and $\bar{a}, \bar{b}, \bar{c}$ are the images of maps $a, b, c$ under the quotient functor $\mathfrak{Z} \rightarrow \mathfrak{U}$ respectively.

In the following, $\overline{\mathfrak{X}}$ denotes the subcategory of $\mathfrak{U}$ consisting of objects $X \in \mathfrak{X}$, for the subcategory $\mathfrak{X}$ satisfying $\mathfrak{D} \subset \mathfrak{X} \subset \bigcap_{i=1}^{n-1} \mathfrak{D}[-i]^{\perp}$.

Lemma 4.1 [8] For any $X \in \mathfrak{Z}$ and $i \geq 1$, there exists a triangle

$$
C_{i} \stackrel{\beta_{X}^{(i)}}{\longrightarrow} X\langle i\rangle \stackrel{\gamma_{X}^{(i)}}{\longrightarrow} X[i] \longrightarrow C_{i}[1]
$$

in $\mathfrak{C}$ with $C_{i} \in \mathfrak{D} * \mathfrak{D}[1] * \cdots * \mathfrak{D}[i-1]$ and with $\beta_{X}^{(i)}$ being $\mathfrak{D}$-epic.

Lemma 4.2 If $(\mathfrak{X}, \mathfrak{Y}[i])$ is a torsion pair for $1 \leq i \leq n-1$ and $\mathfrak{D} \subset \mathfrak{X} \subset \bigcap_{i=1}^{n-1} \mathfrak{D}[-i]^{\perp}$,

then $\mathfrak{D} \subset \mathfrak{Y} \subset \bigcap_{i=1}^{n-1} \mathfrak{D}[-i]^{\perp}$.

Proof: Noting that $\mathfrak{Y}=\mathfrak{X}[-i]^{\perp}$ for $1 \leq i \leq n-1$. Since $\mathfrak{X} \subset \bigcap_{i=1}^{n-1} \mathfrak{D}[-i]^{\perp}=\bigcap_{i=1}^{n-1} \perp \mathfrak{D}[i]$,

we have $\operatorname{Hom}(\mathfrak{X}, \mathfrak{D}[i])=0$, and then

$\operatorname{Hom}(\mathfrak{X}[-i], \mathfrak{D})=0$ or $1 \leq i \leq n-1$. Therefore

$\mathfrak{D} \subset \bigcap_{i=1}^{n-1} \mathfrak{X}[-i]^{\perp}=\mathfrak{Y}$. Since $\mathfrak{D} \subset \mathfrak{Y}, \mathfrak{D}[-i] \subset \mathfrak{X}[-i]$, we have that

$\bigcap_{i=1}^{n-1} \mathfrak{X}[-i]^{\perp} \subset \bigcap_{i=1}^{n-1} \mathfrak{D}[-i]^{\perp}$. Thus we have

$\mathfrak{D} \subset \mathfrak{Y} \subset \bigcap_{i=1}^{n-1} \mathfrak{D}[-i]^{\perp}$.

Lemma 4.3 Let $X$ and $Y$ be two objects in $\mathfrak{Z}$. Then $\operatorname{Hom}(X, Y[i])=0$ for $1 \leq i \leq n-1$ if and only if
$\operatorname{Hom}(X, Y\langle i\rangle)=0$ for $1 \leq i \leq n-1$.

Proof: By Lemma 4.1, we have an exact sequence

$$
\begin{aligned}
& \mathfrak{C}\left(X, C_{i}\right) \stackrel{\mathfrak{C}\left(X, \beta_{Y}^{(i)}\right)}{\longrightarrow} \mathfrak{C}(X, Y\langle i\rangle) \\
& \rightarrow \mathfrak{C}(X, Y[i]) \rightarrow \mathfrak{C}\left(X, C_{i}[1]\right)
\end{aligned}
$$

where $X \in \mathfrak{Z}$ and $\beta_{Y}^{(i)}$ is a right $\mathfrak{D}$-approximation. Since

$C_{i} \in \mathfrak{D} * \mathfrak{D}[1] * \cdots * \mathfrak{D}[i-1]$ and

$\mathfrak{C}(\mathfrak{Z}, \mathfrak{D} * \mathfrak{D}[1] * \cdots * \mathfrak{D}[i-1])=0$, we have

$\mathfrak{C}\left(X, C_{i}\right)=0$ and $\mathfrak{C}\left(X, C_{i}[1]\right)=0$ for $1 \leq i \leq n-1$. Then $\mathfrak{C}(X, Y[i])=0$ if and only if $\mathfrak{C}(X, Y\langle i\rangle)=0$ for $1 \leq i \leq n-1$.

Theorem 4.4 Let $\mathfrak{X}$ be a subcategory of $\mathfrak{C}$ satisfying $\mathfrak{D} \subset \mathfrak{X} \subset \bigcap_{i=1}^{n-1} \mathfrak{D}[-i]^{\perp}$. Then $(\mathfrak{X}, \mathfrak{Y}[i])$ is a torsion pair with $\mathfrak{I}(\mathfrak{X})$ in $\mathfrak{C}$ for $1 \leq i \leq n-1$ if and only if $(\overline{\mathfrak{X}}, \overline{\mathfrak{Y}}\langle i\rangle)$ is a torsion pair with $\mathfrak{I}(\mathfrak{X})$ in $U$ for $1 \leq i \leq n-1$.

Proof: Noting that $\mathfrak{U}$ is a triangulated category with shift functor $\langle i\rangle$, we suppose that $(\mathfrak{X}, \mathfrak{Y}[i])$ is a torsion pair $1 \leq i \leq n-1$. It follows from Lemma 4.2 that $\mathfrak{D} \subset \mathfrak{Y} \subset \bigcap_{i=1}^{n-1} \mathfrak{D}[-i]^{\perp}$. By Lemma 4.3, we have

$\operatorname{Hom}_{\mathfrak{U}}(\mathfrak{X}, \mathfrak{Y}\langle i\rangle)=0$ for $1 \leq i \leq n-1$. For any $Z \in \mathfrak{Z}$, there is a triangle

$$
X \rightarrow Z \rightarrow Y[i] \rightarrow X[1]
$$

where $X \in \mathfrak{X}$ and $Y[i] \in \mathfrak{Y}[i]$ as $(\mathfrak{X}, \mathfrak{Y}[i])$ is a torsion pair in $\mathfrak{C}$. Since all of $X, Y, Z$ are in $\mathfrak{Z}$, there is a triangle

$$
X \rightarrow Z \rightarrow Y\langle i\rangle \rightarrow X\langle i\rangle
$$

in $\mathfrak{U}$. Therefore, $\mathfrak{U} \subset \overline{\mathfrak{X}} * \overline{\mathfrak{Y}}\langle i\rangle$. Hence $(\overline{\mathfrak{X}}, \overline{\mathfrak{Y}}\langle i\rangle)$ is a torsion pair in $\mathfrak{U}$.

Conversely, we suppose $(\overline{\mathfrak{X}}, \overline{\mathfrak{Y}}\langle i\rangle)$ is a torsion pair for $1 \leq i \leq n-1$. By Lemma 4.3, we have $\operatorname{Hom}(\mathfrak{X}, \mathfrak{Y}[i])=0$ for $1 \leq i \leq n-1$. For any $Z \in \mathfrak{Z}$, there is a triangle in $\mathfrak{U}$ :

$$
X \rightarrow Z \rightarrow Y\langle i\rangle \rightarrow X\langle 1\rangle
$$

where $X \in \mathfrak{X}$ and $Y \in \mathfrak{X}[-i]^{\perp}$ for $1 \leq i \leq n-1$ by Lemma 4.3. Then there is a triangle

$$
X \rightarrow Z^{\prime} \rightarrow Y[i] \rightarrow X[1]
$$

in $\mathfrak{C}$ such that $Z \cong Z^{\prime}$ in $\mathfrak{U}$. Hence $Z \cong Z^{\prime}$ in $\mathfrak{C}$ up to direct summands of $\mathfrak{D}$. Thus $\mathfrak{Z}$ is a subcategory of $\mathfrak{X} * \mathfrak{Y}[i]$. Since there is a triangle in $\mathfrak{C}$ for any $Z \in \mathfrak{Z}:$

$$
Z[i-1] \rightarrow D[i-1] \rightarrow Z\langle i\rangle \rightarrow Z[i]
$$

where $Z\langle i\rangle \in \mathfrak{Z}$ and $i \geq 1$, we have

$$
\mathfrak{C}=\mathfrak{Z} * \mathfrak{D}[i] \subset \mathfrak{X} * \mathfrak{Y}[i] * \mathfrak{D}[i]=\mathfrak{X} * \mathfrak{Y}[i] \text {. }
$$


Therefore $(\mathfrak{X}, \mathfrak{Y}[i])$ is a torsion pair in $\mathfrak{C}$ for $1 \leq i \leq n-1$.

Finally, we have $\mathfrak{I}(\overline{\mathfrak{X}})=\overline{\mathfrak{X}} \cap \overline{\mathfrak{Y}}=\overline{\mathfrak{X} \cap \mathfrak{Y}}=\overline{\mathfrak{I}(\mathfrak{X})}$.

Corollary 4.5 Let $\mathfrak{X}$ be a subcategory of $\mathfrak{C}$ satisfying $\mathfrak{D} \subset \mathfrak{X} \subset \bigcap_{i=1}^{n-1} \mathfrak{D}[-i]^{\perp}$, then we have the following:

1) $(\mathfrak{X}, \mathfrak{Y}[i])$ is a rigid torsion pair in $\mathfrak{C}$ for $1 \leq i \leq n-1$ if and only if $\mathfrak{C}(\overline{\mathfrak{X}}, \overline{\mathfrak{Y}}\langle i\rangle)$ is a rigid torsion pair in $\mathfrak{U}$ for $1 \leq i \leq n-1$.

2) $(\mathfrak{X}, \mathfrak{Y}[i])$ is a $n$-cluster tilting torsion pair in $\mathfrak{C}$ for $1 \leq i \leq n-1$ if and only if $(\overline{\mathfrak{X}}, \overline{\mathfrak{Y}}\langle i\rangle)$ is a $n$-cluster tilting torsion pair in $\mathfrak{U}$ for $1 \leq i \leq n-1$.

3) $(\mathfrak{X}, \mathfrak{Y}[i])$ is a maximal rigid torsion pair in $\mathfrak{C}$ for $1 \leq i \leq n-1$ if and only if $(\overline{\mathfrak{X}}, \overline{\mathfrak{Y}}\langle i\rangle)$ is a maximal rigid torsion pair $\mathfrak{U}$ for $1 \leq i \leq n-1$.

Proof: 1) By Corollary 3.6, we only need to prove $\mathfrak{I}(\mathfrak{X})=\mathfrak{X}$ if and only if $\mathfrak{I}(\overline{\mathfrak{X}})=\overline{\mathfrak{X}}$. By Theorem 4.4 we have $\mathfrak{I}(\overline{\mathfrak{X}})=\overline{\mathfrak{I}(\mathfrak{X})}=\overline{\mathfrak{X}}$.

2) It follows from Theorem 4.9 in [8] that we have a one-one correspondence between $n$-cluster tilting subcategories of $\mathfrak{C}$ containing $\mathfrak{D}$ and $n$-cluster tilting subcategories of $\mathfrak{U}$.

3) It is obvious that $\mathfrak{X}[i] \subseteq \mathfrak{Y}[i]$ if and only if $\mathfrak{X}\langle i\rangle \subseteq \mathfrak{Y}\langle i\rangle$ for $1 \leq i \leq n-1$. Assume that,

$M[i-1] \in \mathfrak{X}[i-1] * \mathfrak{X}[i]$ while $i \geq 1$ for any rigid object $M$ in $\mathfrak{C}$. For any rigid object $Y$ in $\mathfrak{U}$, we have that $Y$ is also rigid in $\mathfrak{C}$. Then there is a triangle

$$
X[i-1] \rightarrow X^{\prime}[i-1] \rightarrow Y[i-1] \rightarrow X[i]
$$

in $\mathfrak{C}$, where $X, X^{\prime} \in \mathfrak{X}$ and $i \geq 1$. Thus there is a triangle

$$
X\langle i-1\rangle \rightarrow X^{\prime}\langle i-1\rangle \rightarrow Y\langle i-1\rangle \rightarrow X\langle i\rangle
$$

in $\mathfrak{U}$ with $i \geq 1$. Therefore,

$$
Y\langle i-1\rangle \in \overline{\mathfrak{X}}\langle i-1\rangle * \overline{\mathfrak{X}}\langle i\rangle .
$$

It follows from Corollary 3.3 that $(\overline{\mathfrak{X}}, \overline{\mathfrak{Y}}\langle i\rangle)$ is a maximal rigid torsion pair in $\mathfrak{U}$ for $1 \leq i \leq n-1$.

Conversely, $Y\langle i-1\rangle \in \overline{\mathfrak{X}}\langle i-1\rangle * \overline{\mathfrak{X}}\langle i\rangle$ while $i \geq 1$ for any rigid object $Y$ in $\mathfrak{U}$. For any rigid object $M$ in $\mathfrak{C}$, there is a triangle

$$
Z[i-1] \rightarrow M[i-1] \rightarrow D[i] \rightarrow Z[i]
$$

in $\mathfrak{C}$, where $Z \in \mathfrak{Z}, D \in \mathfrak{D}$ and $i \geq 1$. Applying $\operatorname{Ext}^{1}(D,-)$ to this triangle we obtain that $Z[i-1]$ is a rigid object for $i \geq 1$. Then $Z\langle i-1\rangle$ is a rigid object in $\mathfrak{U}$ for $i \geq 1$. And thus there is a triangle

$$
X\langle i-1\rangle \rightarrow Z\langle i-1\rangle \rightarrow X^{\prime}\langle i\rangle \rightarrow X\langle i\rangle
$$

in $\mathfrak{U}$, where $X, X^{\prime} \in \mathfrak{X}$. Then there is a triangle

$$
X[i-1] \rightarrow Z^{\prime}[i-1] \rightarrow X^{\prime}[i] \rightarrow X[i]
$$

in $\mathfrak{C}$ such that $Z^{\prime}$ is isomorphic to $Z$ up to direct summands in $\mathfrak{D}$. Since $\operatorname{Hom}(\mathfrak{X}, \mathfrak{X}[i])=0$ for $1 \leq i \leq n-1$, it is easy to see that $\mathfrak{X} * \mathfrak{X}[i]$ is closed under extensions for $1 \leq i \leq n-1$.

Therefore $Z[i-1] \in \mathfrak{X}[i-1] * \mathfrak{X}[i]$ and then $M[i-1] \in \mathfrak{X}[i-1] * \mathfrak{X}[i] * \mathfrak{D}[i]=\mathfrak{X}[i-1] * \mathfrak{X}[i]$ because $\mathfrak{D}[i] \subseteq \mathfrak{X}[i-1] * \mathfrak{X}[i]$ by $(*)$. So we have that $(\mathfrak{X}, \mathfrak{Y}[i])$ is a maximal rigid torsion pair in $\mathfrak{C}$ for $1 \leq i \leq n-1$.

\section{Acknowledgement}

Supported by the National Natural Science Foundation of China (Grant No. 10971172, 11271119) and the Natural Science Foundation of Beijing (Grant No. 1122002).

\section{REFERENCES}

[1] A. Beilinson, J. Bernstein and P. Deligne, "Faisceaux Pervers,” Asterisque 100, 1982.

[2] A. Beligiannis and I. Reiten, "Homological and Homotopical Aspects of Torsion Theories,” 2007. http://www.math.uoi.gr/ abeligia/torsion.pdf

[3] A. L. Gorodentsev and A. N. Rudakov, "Exceptional Vector Bundles on Projective Spaces," Duke Mathematical Journal, Vol. 54, No. 1, 1987, pp. 115-130. doi:10.1215/S0012-7094-87-05409-3

[4] S. Fomin and A. Zelevinsky, "Cluster Algebras I. Foundations,” Journal of American Mathematical Society, Vol. 15, No.2, 2002, pp. 497-529. doi:10.1090/S0894-0347-01-00385-X

[5] S. Fomin and A. Zelevinsky, "Cluster Algebras II. Finite Type Classification,” Inventiones Mathematicae, Vol. 154, No. 1, 2003, pp. 63-121. doi:10.1007/s00222-003-0302-y

[6] A. Buan, R. Marsh, M. Reineke, I. Reiten and G. Todorov, “Tilting Theory and Cluster Combinations," Advances in Mathematics, Vol. 204, No. 2, 2006, pp. 572-618. doi:10.1016/j.aim.2005.06.003

[7] C. Geiss, B. Leclerc and J. Schroer, "Rigid Modules over Preprojective Algebras," Inventiones Mathematicae, Vol. 165, No. 3, 2006, pp. 589-632. doi:10.1007/s00222-006-0507-y

[8] M. Kontsevich, “Triangulated Categories and Geometry," The École Normale Supérieure, Paris, 1998.

[9] O. Iyama and Y. Yoshino, "Mutations in Triangulated Categories and Rigid Cohen-Macaulay Modules,” Inventiones mathematicae, Vol. 172, No. 1, 2008, pp. 117-168. doi:10.1007/s00222-007-0096-4

[10] Y. Zhou and B. Zhu, "Mutation of Torsion Pairs in Triangulated Categories and Its Geometric Realization," arXiv.org, Los Alamos, 2011.

[11] M. Auslander and S. O. Smalø, "Almost Split Sequences in Subcategories,” Journal of Algebra, Vol. 69, No. 2, 1981, pp. 426-454. doi:10.1016/0021-8693(81)90214-3

[12] B. Keller and I. Reiten, "Cluster-Tilted Algebras Are Gorenstein and Stably Calabi-Yau,” Advances in Mathe- 
matics, Vol. 211, No. 1, 2007, pp. 123-151.

doi:10.1016/j.aim.2006.07.013

[13] Y. Zhou and B. Zhu, "Cluster Combinatorics of d-Cluster
Categories,” Journal of Algebra, Vol. 321, No. 10, 2009, pp. 2898-2915. doi:10.1016/j.jalgebra.2009.01.032 